\title{
Alien Crosstalk Cancellation for Multipair Digital Subscriber Line Systems
}

\author{
George Ginis and Chia-Ning Peng \\ Broadband Communications Group, Texas Instruments Incorporated, 2043 Samaritan Drive, San Jose, CA, USA
}

Received 30 November 2004; Revised 18 February 2005; Accepted 4 May 2005

\begin{abstract}
An overview of alien crosstalk cancellation for DSL systems with multiple pairs is here presented. It is shown that when a common crosstalk source affects the receivers of multiple pairs, the noise exhibits a certain correlation among the pairs. In a DMT system, the frequency-domain noise samples are most strongly correlated between pairs when they correspond to the same tone. Thus, noise decorrelation algorithms applied independently for each tone can provide significant performance enhancements. Three possible methods are described for noise decorrelation, one is suitable for two-sided coordination and two are suited for receiver coordination among the pairs. It is theoretically proven that the data-rate performance of these three methods is identical from the perspective of the sum rate over all pairs. Simulation results corresponding to an ADSL2+ two-pair system with a $\mathrm{T} 1$ disturber are presented to illustrate the noise correlation property and to indicate the potential performance bene-
\end{abstract} fits.

Copyright @ 2006 Hindawi Publishing Corporation. All rights reserved.

\section{INTRODUCTION}

Digital subscriber line (DSL) transmission is typically constrained by crosstalk interference. As DSL technology advances and processing power increases, the interest in techniques for crosstalk reduction has increased. Most of these techniques are based on multiple-input multiple-output (MIMO) system representations, and it is common in the DSL literature to refer to them as vectoring methods and to describe a DSL system employing such methods as a vectored system. This paper gives an overview of a specific class of vectoring methods that aim to suppress interference arising from crosstalk sources that lie outside the vectored system. The effect of such sources is typically referred to as alien or out-of-domain crosstalk. These concepts are further explained in the paragraphs that follow.

Vectored systems are comprised of multiple twisted pairs and the corresponding transceiver modules. The multiple twisted pairs are represented as a MIMO channel. In a vectored system, joint signal processing (or MIMO) techniques are employed on one or both sides of the MIMO channel. Vectoring techniques that require joint signal processing at both sides fall under the category of two-sided coordination. Vectoring techniques that only require joint signal processing at one side are described as coordinated reception or coordinated transmission, depending on the side where the joint signal processing takes place.
An alternative categorization of vectoring methods relates to the type of crosstalk that is being cancelled. The crosstalk interference affecting transmission of information on a pair belonging to the vectored system can originate from two kinds of sources. Crosstalk may originate from a transmitter belonging to the vectored system, or it may originate from a transmitter outside the vectored system. The first kind is defined as in-domain crosstalk, while the second kind is defined as alien or out-of-domain crosstalk.

One of the earliest references to MIMO methods for DSL is [1], where both transmitter and receiver methods were described. The concept of alien crosstalk cancellation was described extensively in $[2,3]$. This early research was later followed by work focusing on the discrete multitone (DMT) modulation method. Methods for coordinated reception/transmission of in-domain crosstalk cancellation were described in $[4,5]$, which involved MIMO decision feedback at the receiver, and MIMO precoding at the transmitter. Two-sided coordination for in-domain crosstalk cancellation was proposed in [6]. References $[7,8]$ showed that significant performance benefits are still possible with simplified vectoring techniques that achieve partial cancellation. Linear MIMO signal processing was shown in $[9,10]$ to be near-optimal for in-domain crosstalk cancellation. A general theory of MIMO for DSL and an overview of its applications are given in [11]. An alternative approach towards crosstalk cancellation which exploits the common-mode signal of DSL 


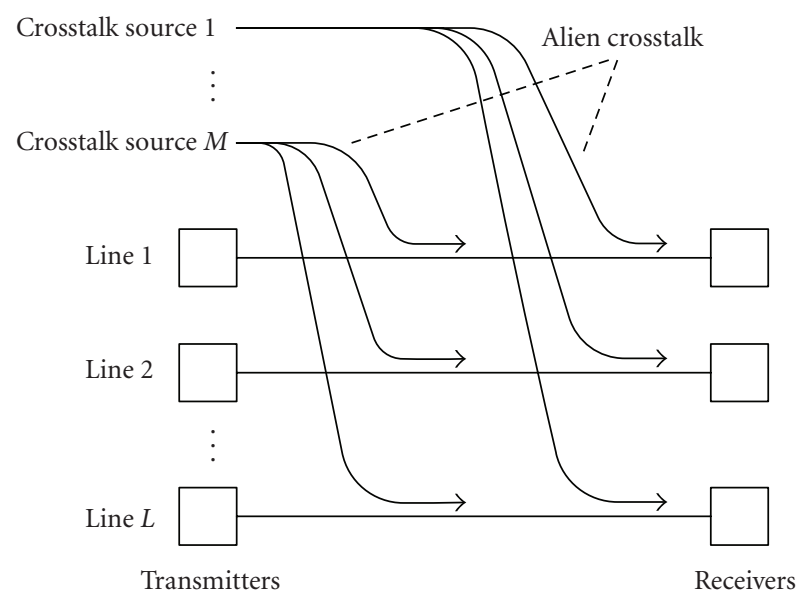

FIGURE 1: DSL crosstalk environment.

pairs and which does not rely on joint signal processing of signals from multiple pairs was presented in [12].

Vectoring methods have attracted significant interest from the DSL industry. Such methods have been presented and discussed within DSL standardization bodies such as the Network Access Interfaces (NAI) Committee of the Alliance for Telecommunications Industry Solutions (ATIS). These discussions have partly been motivated by the application of DSL bonding, where multiple pairs are used to transport a single data stream, and which offers the possibility to implement two-sided coordination. Contribution [13] presented information on the concept of alien crosstalk cancellation using two-sided coordination. The benefits of vectoring were analyzed using real measured channels in [14]. Alien crosstalk cancellation is also described in detail in [15].

This paper presents the theory of alien crosstalk cancellation, and gives an overview of such MIMO methods. The system model is given in Section 2, where the assumption of DMT transmission is made and the property of noise correlation among tones is illustrated. Section 3 describes a number of approaches for alien crosstalk cancellation (also referred to as MIMO noise decorrelation), and shows their equivalence from an information-theory standpoint. The approach of receiver coordination with noise prediction for DMT has not been previously proposed in the literature. Section 4 shows simulation results for alien crosstalk cancellation, and Section 5 draws some final conclusions.

The following notation is used in the rest of this paper. Bold letters indicate vectors, lowercase is used for timedomain signals, and uppercase is used for frequency-domain signals. The superscripts $T$ and $*$ denote the transpose and conjugate transpose operations correspondingly.

\section{SYSTEM MODEL}

\subsection{Channel model}

The DSL environment for a vectored system is shown in Figure 1. It is assumed that the vectored system consists of
$L$ twisted pairs, and that crosstalk originates from $M$ sources that lie outside the vectored system. These sources may give rise to either of far-end crosstalk (FEXT) near-end crosstalk (NEXT), but this fact has no consequence for the analysis that follows. The important observation is that alien crosstalk affects the vectored pairs in a common way. This implies that the received noise has a certain degree of correlation among the vectored pairs. Exploiting this noise correlation is the fundamental concept for alien crosstalk cancellation.

Using vector notation, the output signal on pair $i$ can be expressed as

$$
\mathbf{y}_{i}=H_{i}^{c} \mathbf{x}_{i}^{p}+\mathbf{n}_{i}+\sum_{k=1}^{M} A_{i, k}^{c} \mathbf{z}_{k}^{p}
$$

where $\mathbf{y}_{i}$ is a column vector containing a block of $N$ received samples, $\mathbf{x}_{i}^{p}$ is $(N+\nu) \times 1$ and holds the transmitted samples on pair $i, \mathbf{n}_{i}$ is $N \times 1$ and contains the Gaussian-distributed noise samples received on pair $i$, and $\mathbf{z}_{k}^{p}$ is $(N+\xi) \times 1$ and holds the transmitted samples of crosstalk source $k$. The samples contained in these vectors are ordered from the most recent to the least recent. The noise correlation matrix can be assumed to be $R_{\mathbf{n}_{i} \mathbf{n}_{i}}=I$ with no loss of generality. The matrix $H_{i}^{c}$ is $N \times(N+v)$ and represents the convolution matrix corresponding to the channel of pair $i$. The matrix $A_{i, k}^{c}$ is $N \times(N+\xi)$ and represents the convolution matrix of the crosstalk coupling channel from source $k$ to victim pair $i$. The parameters $v$ and $\xi$ denote the length (in number of samples) of the nonzero part of the impulse response of the direct channel and of the alien crosstalk coupling correspondingly. The sampling rates and sampling times are assumed to be identical for all pairs. It is noted that the direct and the indirect channels are here assumed to include the time-domain filtering of the transmitter and receiver modems.

\subsection{DMT transmission and synchronization}

DMT transmission is here considered, since it is by far the most popular transmission method in DSL. It is assumed that each DMT symbol includes both a cyclic prefix $(\mathrm{CP})$ and a cyclic suffix (CS) [16]. It is also assumed that timing advance is employed at the customer premises' modems as defined in the very-high-speed digital subscriber line (VDSL) recommendation [17].

A timing diagram is shown in Figure 2 to illustrate the concept of synchronized DMT transmission among multiple pairs. The time scale corresponds to the vertical axis. Two pairs are assumed and both transmission directions are considered. LT-TX1 and LT-TX2 indicate the transmitters at the line termination (LT) or central office's side for the two pairs. NT-TX1 and NT-TX2 indicate the transmitters at the network termination (NT) or customer premises' side for the two pairs. The corresponding receivers are denoted as NT-RX1, NT-RX2, LT-RX1, and LT-RX2. The transmission is shown for the duration of a single DMT block. The following example assumptions are made. The channel memory is equal 


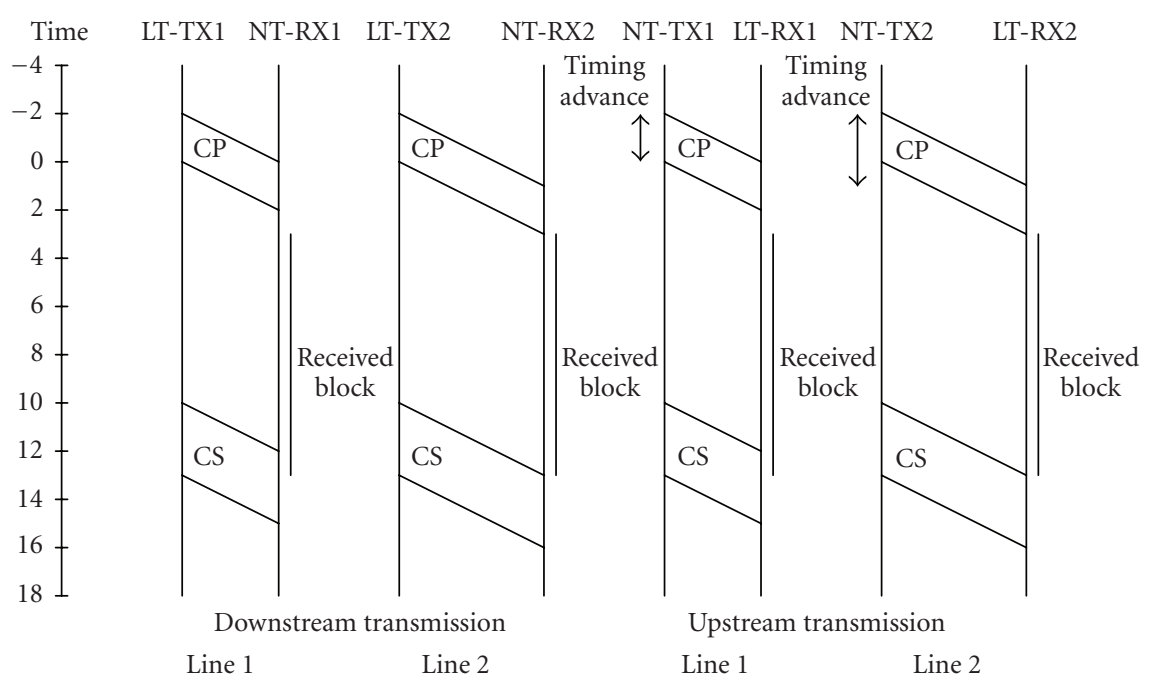

Figure 2: Example of synchronized lines.

to 2 units of time, and the CP is set equal to 2 units of time. The propagation delay of pair 1 is 2 units of time, and the propagation delay of pair 2 is 3 units of time. The maximum propagation delay is equal to 3 units of time, so the CS is also set to 3 units of time. The timing advance at the NT side equals the propagation delay of the corresponding pair.

In the above example, the block transmission of the main part of the DMT symbol starts at time 0 for the LT transmitters. The transmission of the CP starts at time -2 . The timing advance at the NT side means that the block transmission at the NT side is advanced by an amount of time equal to the propagation delay of the line. This results in DMT symbols at the NT side starting transmission at the same time as those at the LT side. Evidently, the DMT blocks are synchronized across all transmitters.

Next, the receiver timing is examined. It is a requirement for the DMT blocks to be synchronized across the receivers, in order to fully exploit noise correlation among the receivers. The existence of the cyclic suffix makes this possible: the thick vertical line segments show the block of samples that are extracted at the receivers. For NT-RX2, the received block includes all the samples of the main part of the DMT symbol. For NT-RX1, the received block includes part of the CS, in order to coincide in time with the received block of NT-RX2. Similarly, the received blocks at the LT side are chosen to coincide in time. The inclusion of part of the CS implies only a phase change for the channel model. A mathematical representation of this scheme is given next.

The inclusion of the CP and the CS can be expressed as

$$
\mathbf{x}_{i}^{p}=P_{i} \mathbf{x}_{i}
$$

where $\mathbf{x}_{i}$ is an $N \times 1$ vector containing the DMT block samples to be transmitted excluding the CP and the CS. The parameter $N$ is chosen to be equal to twice the number of DMT tones. The matrix $P_{i}$ is $(N+v) \times N$ and models the addition of the CP and CS. Letting the length of the CP be equal to $\nu$, the length of the CS that is included in the received block be equal to $\mu_{i}$, and with the assumption that $\mu_{i}<v$, there exists

$$
P_{i}=\left[\begin{array}{c}
O_{\mu_{i} \times\left(N-\mu_{i}\right)} I_{\mu_{i} \times \mu_{i}} \\
I_{N \times N} \\
I_{\left(\nu-\mu_{i}\right) \times\left(\nu-\mu_{i}\right)} O_{\left(\nu-\mu_{i}\right) \times\left(N-\nu+\mu_{i}\right)}
\end{array}\right] .
$$

Additionally, the FFT and IFFT operations are represented as

$$
\begin{aligned}
& \mathbf{Y}_{i}=Q \mathbf{y}_{i} \\
& \mathbf{x}_{i}=Q^{*} \mathbf{X}_{i},
\end{aligned}
$$

where $\mathbf{Y}_{i}$ is the FFT output at the receiver, and $\mathbf{X}_{i}$ is the IFFT input at the transmitter. The matrices $Q$ and $Q^{*}$ correspond to the FFT and IFFT transforms, respectively.

From (1), (2), (4), a new expression is obtained for the received samples of user $i$ at the FFT output:

$$
\begin{aligned}
\mathbf{Y}_{i} & =Q H_{i}^{c} P_{i} Q^{*} \mathbf{X}_{i}+Q \mathbf{n}_{i}+\sum_{k=1}^{M} Q A_{i, k}^{c} \mathbf{z}_{k}^{p} \\
& =Q H_{i} Q^{*} \mathbf{X}_{i}+\mathbf{N}_{i}^{\prime}+\sum_{k=1}^{M} \mathbf{A}_{i, k} \\
& =\Lambda_{i} \mathbf{X}_{i}+\mathbf{N}_{i}^{\prime}+\mathbf{A}_{i} .
\end{aligned}
$$

Note that $H_{i}=H_{i}^{c} P_{i}$ is a circulant matrix, which makes $\Lambda_{i}=$ $\mathrm{QH}_{i} \mathrm{Q}^{*}$ a diagonal matrix. The correlation matrix of the new noise term is $R_{\mathbf{N}_{i}^{\prime} \mathbf{N}_{i}^{\prime}}=I$. The choice of $\mu_{i}$ in the definition of $P_{i}$ depends on the timing of the received block. It can be shown that this choice only affects the phase of the diagonal elements of $\Lambda_{i}$. The next subsection investigates the term $\mathbf{A}_{i}$, which represents the alien crosstalk. 


\subsection{Alien crosstalk correlation}

In (7), the alien crosstalk for user $i$ is defined as

$$
\begin{aligned}
\mathbf{A}_{i} & =\sum_{k=1}^{M} \mathbf{A}_{i, k} \\
& =\sum_{k=1}^{M} Q A_{i, k}^{c} \mathbf{z}_{k}^{p} .
\end{aligned}
$$

With the assumption that crosstalk sources are independent, the correlation matrix of alien crosstalk of user $i$ is

$$
R_{\mathbf{A}_{i} \mathbf{A}_{i}}=\sum_{k=1}^{M} Q A_{i, k}^{c} E\left(\mathbf{z}_{k}^{p} \mathbf{z}_{k}^{p *}\right) A_{i, k}^{c *} Q^{*},
$$

while the correlation matrix of alien crosstalk of user $i$ with that of user $j$ is

$$
R_{\mathbf{A}_{i} \mathbf{A}_{j}}=\sum_{k=1}^{M} Q A_{i, k}^{c} E\left(\mathbf{z}_{k}^{p} \mathbf{z}_{k}^{p *}\right) A_{j, k}^{c *} Q^{*} .
$$

There is a special case that deserves some attention. Setting $\xi=v$ and $z_{N-k}=z_{-k}$, for $k=1, \ldots, v$, results in

$$
\mathbf{z}_{k}^{p}=P \mathbf{z}_{k}
$$

where $P$ is as in (3) with $\mu_{i}=0$. These conditions correspond to the special case where the alien crosstalk sources are DMT transmitters with the same sampling rates, same block sizes, and same CP and CS as the vectored system. Also, the transmission of the alien crosstalk sources must be such that the DMT symbol boundaries are synchronized with the received DMT symbols of the vectored system. This special case does not correspond to a practical situation, but it does provide some intuition.

Assuming that the transmitted samples of the alien crosstalk are white, there exists $E\left(\mathbf{z}_{k} \mathbf{z}_{k}^{*}\right)=\mathcal{E}_{z, k} I$. (Note that any transmitter spectrum shaping of the alien crosstalk sources can be incorporated in the alien crosstalk coupling.) Then, the correlation matrix of the alien crosstalk of user $i$ with that of user $j$ becomes

$$
\begin{aligned}
R_{\mathbf{A}_{i} \mathbf{A}_{j}} & =\sum_{k=1}^{M} Q A_{i, k}^{c} P E\left(\mathbf{z}_{k} \mathbf{z}_{k}^{*}\right) P^{*} A_{j, k}^{c *} Q^{*} \\
& =\sum_{k=1}^{M} Q A_{i, k} E\left(\mathbf{z}_{k} \mathbf{z}_{k}^{*}\right) A_{j, k}^{*} Q^{*} \\
& =\sum_{k=1}^{M} \mathcal{E}_{z, k} \Lambda_{i, k}^{A} \Lambda_{j, k}^{A *},
\end{aligned}
$$

where $\Lambda_{i, k}^{A}=Q A_{i, k} Q^{*}$ is a diagonal matrix. This expression shows that for this special case, noise correlation exists only when the same tone is examined for different users. In other words, there is no correlation between noise of different users, when that noise corresponds to different tones.

When the above special case does not hold, noise on some tone of one user may be correlated with noise on another tone of a second user. This is attributed to the effect of FFT spreading, and it is known that it can be controlled through appropriate receiver windowing.

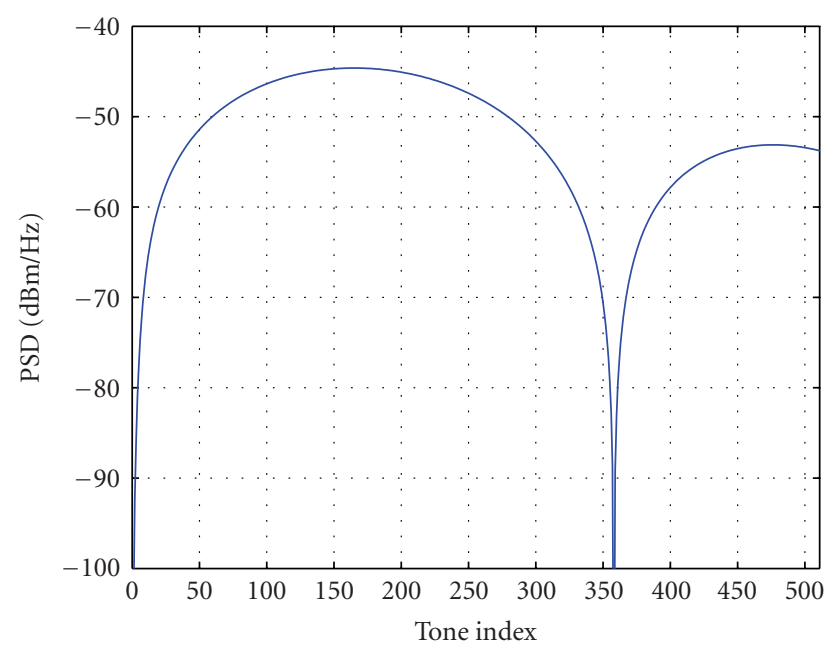

Figure 3: Power spectral density of T1 transmitter.

\subsection{Example}

An example is next presented to illustrate the noise correlation property in practical conditions. It is assumed that there is a single crosstalk source from a T1 transmitter, which affects the downstream receivers of 2 ADSL2+ systems [18]. Other noise sources (e.g., background noise) are here ignored.

The bandwidth of the ADSL2+ system is $2.208 \mathrm{MHz}$, where 512 tones with a $4.3125 \mathrm{kHz}$ spacing are used. The power spectral density (PSD) of the T1 transmitter over this frequency region is shown in Figure 3.

It is assumed that the ADSL2+ receivers are affected by NEXT interference originating from the T1 transmitter. Actual crosstalk measurements were used to calculate the resulting effect, which were obtained through Stanford University. The measurements were made over $300 \mathrm{~m}$ of $26 \mathrm{AWG}$ cable. Two sets of coupling measurements are here used, with set 1 representing the coupling between pairs 2 and 6 , and set 2 representing the coupling between pairs 2 and 11 within a single binder. In what follows, it is assumed that set 1 expresses the coupling between the crosstalk source and vectored pair 1, while set 2 expresses the coupling between the crosstalk source and vectored pair 2 . The magnitude of the NEXT coupling is shown in Figure 4.

For the numerical computations that follow, (9) and (10) are used. The frequency responses of the crosstalk coupling are converted to impulse responses and shortened to capture $99.9 \%$ of the impulse energy, thus determining the elements of $A_{i, k}^{c}$. The $E\left(\mathbf{z}_{k}^{p} \mathbf{z}_{k}^{p *}\right)$ correlation matrix is derived by computing the correlation of the transmitted samples of the crosstalk source. For this computation, the transmit spectrum shaping is modeled through an FIR filter with 81 samples.

Figure 5 shows a curve representing the magnitude of the noise variance of pair 1 , and five curves representing the magnitude of the correlation between noise on a specific tone and other tones of pair 1 . In the five curves representing the 


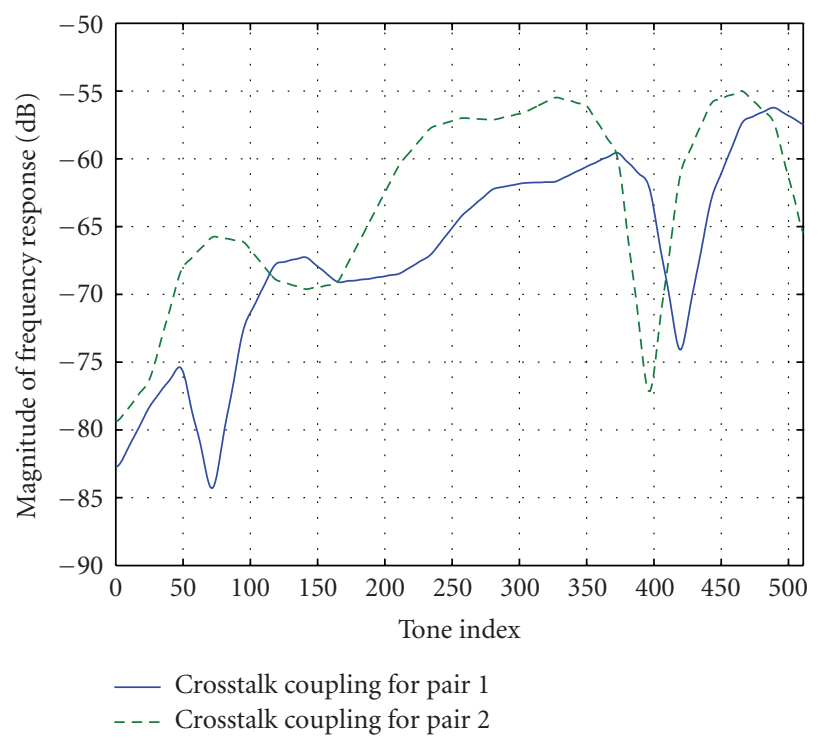

FIgURE 4: Crosstalk coupling for the 2 vectored pairs.

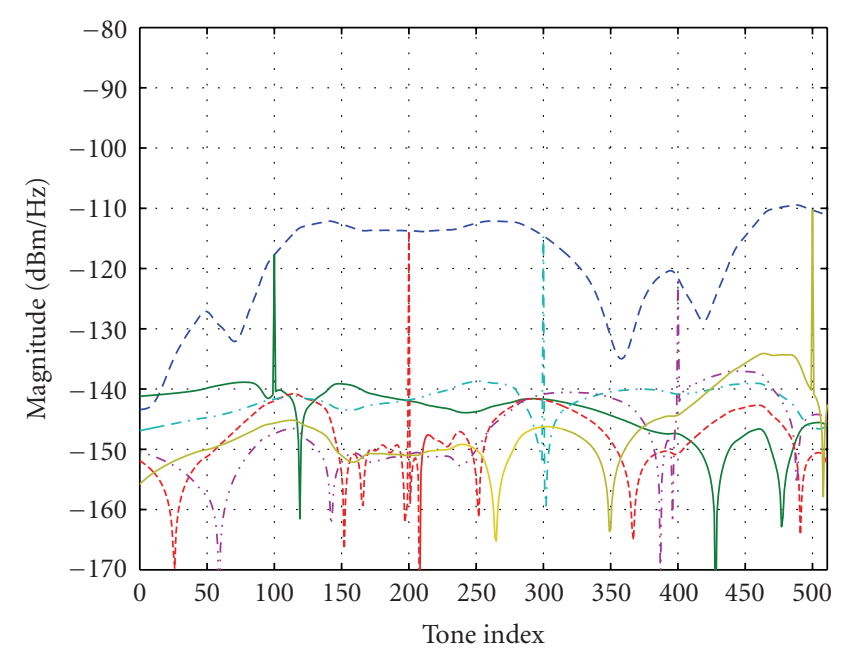

- - - Noise variance across tones

_ Noise correlation between tone 100 and other tones

-... Noise correlation between tone 200 and other tones

. . - Noise correlation between tone 300 and other tones

..... Noise correlation between tone 400 and other tones

- Noise correlation between tone 500 and other tones

FIGURE 5: Noise correlation for user 1.

correlation, the reference is chosen to be tones 100, 200, 300, 400 , and 500, respectively. The noise variance corresponds to the dashed curve, and the other curves can be easily recognized by noting the point where they coincide with the noise variance curve. The main observation from these results is that the noise among tones is indeed correlated to some extent, but that such correlation is relatively weak.

Figure 6 shows similar curves corresponding to user 2 . Again, it is observed that noise correlation between tones is relatively weak.
Finally, Figure 7 presents a curve with the magnitude of the correlation between noise of pair 1 and of pair 2 for the same tone, and five curves with the magnitude of the correlation between noise on a specific tone of pair 1 and noise on tones of pair 2. For the five curves where a specific tone of pair 1 is used as a reference, the tone index is chosen as 100, $200,300,400$, and 500, respectively. It is observed that, on the one hand, the correlation between different tones of different pairs is relatively weak. On the other hand, the correlation between pairs 1 and 2 for the same tone is significant and comparable to the noise variances of the two pairs. This indicates that methods exploiting the noise correlation between pairs within the same tone are of interest, while methods for noise decorrelation among different tones are of much lesser use.

\section{NOISE DECORRELATION}

\subsection{Multiuser model}

Equation (7) is here repeated for convenience,

$$
\mathbf{Y}_{i}=\Lambda_{i} \mathbf{X}_{i}+\mathbf{N}_{i}^{\prime}+\mathbf{A}_{i}
$$

In the above equation, the received frequency-domain samples of user $i$ are given, where vector samples correspond to different tones, and $i=1, \ldots, L$. Since $\Lambda_{i}$ is diagonal, this expression can also be given as

$$
Y_{i, n}=\Lambda_{i, n} X_{i, n}+N_{i, n}^{\prime}+A_{i, n}
$$

where $n=1, \ldots, N$ is the tone index, and where $\mathbf{Y}_{i}=$ $\left[Y_{i, 1} \cdots Y_{i, N}\right]^{T}, \mathbf{X}_{i}=\left[X_{i, 1} \cdots X_{i, N}\right]^{T}, \mathbf{N}_{i}^{\prime}=\left[N_{i, 1}^{\prime} \cdots N_{i, N}^{\prime}\right]^{T}$, $\mathbf{A}_{i}=\left[A_{i, 1} \cdots A_{i, N}\right]^{T}$, and $\Lambda_{i, n}$ are the diagonal elements of $\Lambda_{i}$.

Reorganizing (14) into vectors corresponding to a specific tone, the following expression is obtained:

$$
\mathbf{Z}_{n}=T_{n} \mathbf{W}_{n}+\mathbf{N}_{n}, \quad n=1, \ldots, N
$$

where $\mathbf{Z}_{n}=\left[Y_{1, n} \cdots Y_{L, n}\right]^{T}, \mathbf{W}_{n}=\left[X_{1, n} \cdots X_{L, n}\right]^{T}$, and $\mathbf{N}_{n}=$ $\left[N_{1, n} \cdots N_{L, n}\right]^{T}$. Also, $T_{n}$ is a diagonal matrix with elements $\Lambda_{1, n}, \ldots, \Lambda_{L, n}$. The term $\mathbf{N}_{n}$ includes noise components corresponding to both background noise $N_{i, n}^{\prime}$ and alien crosstalk $A_{i, n}$. In the expression above, $R_{\mathbf{N}_{n} \mathbf{N}_{n}}$ is in general nondiagonal, as explained in Section 2.

\subsection{Two-sided coordination}

Two-sided coordination for DSL has been proposed in several instances. Reference [1] first showed the concept for DSL and presented transmitter and receiver design methods. In [2], the idea of exploiting noise correlation with two-sided coordination was first analyzed. Also, $[13,15]$ described an approach for alien crosstalk cancellation for DMT-based systems with two-sided coordination.

The analysis of this section essentially makes use of the methodology of [19], when applied to DSL. Starting from 


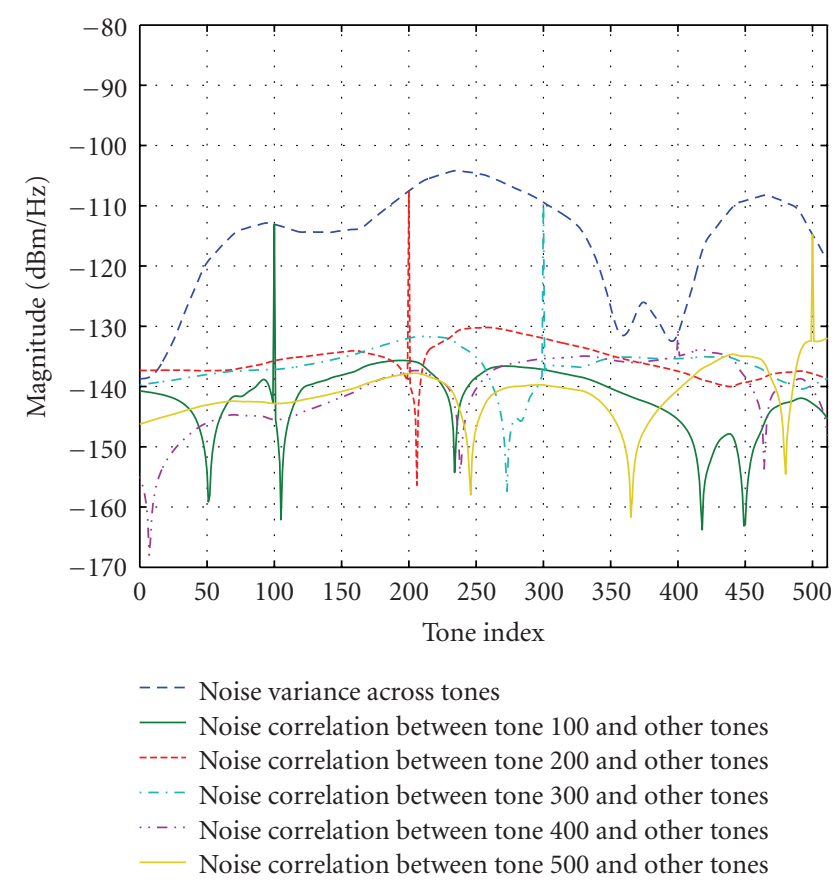

FIGURE 6: Noise correlation for user 2.

(15), it is noted that the noise correlation matrix has the following Cholesky decomposition:

$$
R_{\mathbf{N}_{n} \mathbf{N}_{n}}=G_{n} R_{\mathbf{E}_{n} \mathbf{E}_{n}} G_{n}^{*}
$$

where $G_{n}$ is a lower-triangular matrix with diagonal elements equal to 1 , and $R_{\mathbf{E}_{n} \mathbf{E}_{n}}$ is a diagonal matrix with positive elements.

The singular value decomposition (SVD) of the equivalent channel of tone $n$ after noise decorrelation is expressed as

$$
\begin{aligned}
\widetilde{T}_{n} & =R_{\mathbf{N}_{n} \mathbf{N}_{n}}^{-1 / 2} T_{n} \\
& =R_{\mathbf{E}_{n} \mathbf{E}_{n}}^{-1 / 2} G_{n}^{-1} T_{n} \\
& =U_{n} \Sigma_{n} V_{n}^{*},
\end{aligned}
$$

where $U_{n}$ and $V_{n}$ are unitary matrices, and $\Sigma_{n}$ is diagonal with positive elements in decreasing order. (Square-root factorization other than Cholesky can also be used to obtain the equivalent channel after noise decorrelation.)

Then, channel diagonalization is achieved by the following operations. At the transmitter,

$$
\mathbf{W}_{n}=V_{n} \widetilde{\mathbf{W}}_{n}
$$

where $\widetilde{\mathbf{W}}_{n}$ is the input of the operation. At the receiver,

$$
\widetilde{\mathbf{Z}}_{n}=U_{n}^{*} R_{\mathbf{N}_{n} \mathbf{N}_{n}}^{-1 / 2} \mathbf{Z}_{n}
$$

where $\widetilde{\mathbf{Z}}_{n}$ is the output of the operation.

Combining (15), (19), (20), and (21),

$$
\widetilde{\mathbf{Z}}_{n}=\Sigma_{n} \widetilde{\mathbf{W}}_{n}+\widetilde{\mathbf{N}}_{n}
$$

where $\tilde{\mathbf{N}}_{n}=U_{n}^{*} R_{\mathbf{N}_{n} \mathbf{N}_{n}}^{-1 / 2} \mathbf{N}_{n}$ and $R_{\widetilde{\mathbf{N}}_{n} \tilde{\mathbf{N}}_{n}}=I$, so that the noise samples are no longer correlated.

The transmitter and receiver structures are shown in Figure 8.

Next, it is shown that on a per-tone basis, the maximum achievable data-rate sum with this method is equal to the capacity of the channel. The capacity of the vector channel of (15) is defined as the maximum of the mutual information between $\mathbf{Z}_{n}$ and $\mathbf{W}_{n}$ [21]. With $\mathbf{W}_{n}$ having a Gaussian distribution, the mutual information is expressed as

$$
\begin{aligned}
I\left(\mathbf{W}_{n} ; \mathbf{Z}_{n}\right) & =H\left(\mathbf{Z}_{n}\right)-H\left(\mathbf{Z}_{n} \mid \mathbf{W}_{n}\right) \\
& =\frac{1}{2} \log _{2} \frac{\left|R_{\mathbf{Z}_{n} \mathbf{Z}_{n}}\right|}{\left|R_{\mathbf{N}_{n} \mathbf{N}_{n}}\right|} \\
& =\frac{1}{2} \log _{2} \frac{\left|T_{n} R_{\mathbf{W}_{n} \mathbf{W}_{n}} T_{n}^{*}+R_{\mathbf{N}_{n} \mathbf{N}_{n}}\right|}{\left|R_{\mathbf{N}_{n} \mathbf{N}_{n}}\right|}
\end{aligned}
$$

where $R_{\mathbf{Z}_{n} \mathbf{Z}_{n}}$ and $R_{\mathbf{W}_{n} \mathbf{W}_{n}}$ are the correlation matrices of $\mathbf{Z}_{n}$ and $\mathbf{W}_{n}$, respectively.

The transmitter and receiver operations of (20) and (21) represent 1-1 transformations. Therefore, the mutual information of the channel of (22) equals the mutual information of the original channel,

$$
I\left(\widetilde{\mathbf{W}}_{n} ; \widetilde{\mathbf{Z}}_{n}\right)=I\left(\mathbf{W}_{n} ; \mathbf{Z}_{n}\right)
$$

Finally, the mutual information can be expressed as

$$
\begin{aligned}
I\left(\widetilde{\mathbf{W}}_{n} ; \widetilde{\mathbf{Z}}_{n}\right) & =\frac{1}{2} \log _{2} \frac{\mid R_{\widetilde{\mathbf{Z}}_{n} \widetilde{\mathbf{Z}}_{n} \mid}}{\left|R_{\widetilde{\mathbf{N}}_{n} \tilde{\mathbf{N}}_{n}}\right|} \\
& =\frac{1}{2} \log _{2} \frac{\mid \sum_{n} R_{\widetilde{\mathbf{W}}_{n} \widetilde{\mathbf{W}}_{n}} \sum_{n}^{*}+R_{\widetilde{\mathbf{N}}_{n} \widetilde{\mathbf{N}}_{n} \mid}}{\left|R_{\widetilde{\mathbf{N}}_{n} \tilde{\mathbf{N}}_{n}}\right|},
\end{aligned}
$$

where $R_{\widetilde{\mathbf{Z}}_{n}} \widetilde{\mathbf{Z}}_{n}$ and $R_{\widetilde{\mathbf{W}}_{n}} \widetilde{\mathbf{W}}_{n}$ are the correlation matrices of $\widetilde{\mathbf{Z}}_{n}$ and $\widetilde{\mathbf{W}}_{n}$, respectively. By making use of Hadamard's inequality,

$$
I\left(\widetilde{\mathbf{W}}_{n} ; \widetilde{\mathbf{Z}}_{n}\right) \leq \sum_{k=1}^{L} \frac{1}{2} \log _{2}\left(1+\rho_{n, k}^{2} \boldsymbol{E}_{n, k}\right),
$$

where $\rho_{n, k}$ are the diagonal elements of $\Sigma_{n}$, and $\varepsilon_{n, k}$ are the diagonal elements of $R_{\widetilde{\mathrm{W}}_{n} \widetilde{\mathrm{W}}_{n}}$. Equality holds when the offdiagonal elements of $R_{\widetilde{W}_{n} \widetilde{\mathbf{W}}_{n}}$ equal 0 .

Thus, transmission optimization becomes the problem of power allocation to parallel channels, which has the wellknown water-filling solution [22]. It is clear from above that two-sided coordination is capable of reaching the maximum achievable data rate.

\subsection{Receiver coordination with decision-feedback structure}

Alien crosstalk cancellation with receiver coordination was investigated in [3], where both decision-feedback approaches and noise-predictive approaches were proposed to achieve noise decorrelation. A general framework for MIMO receivers was presented in [20] as the generalized DFE (GDFE). The GDFE can be shown to contain as subcases many popular MIMO techniques. The application of the GDFE for 


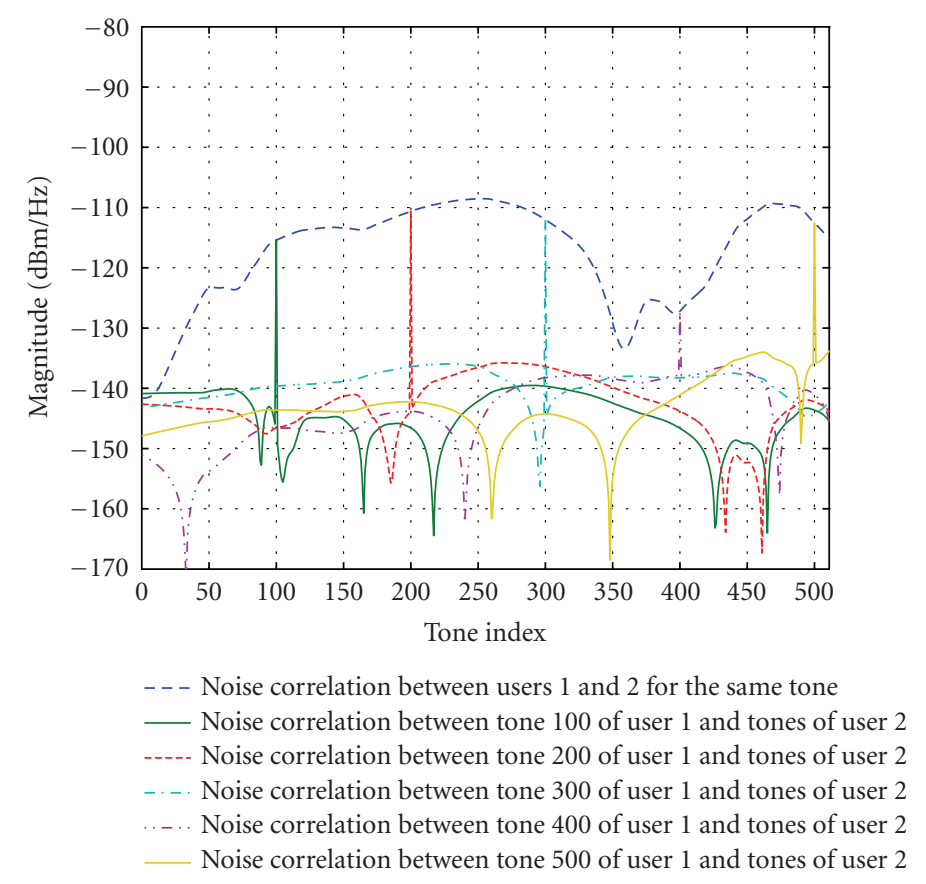

Figure 7: Noise correlation between user 1 and user 2.

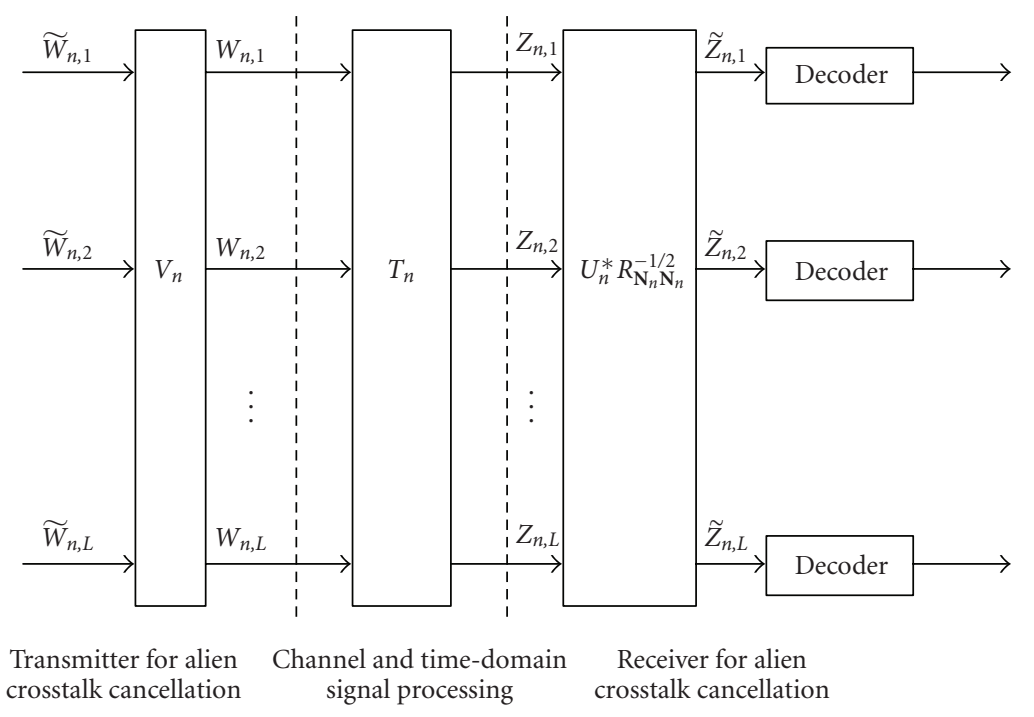

FIGURE 8: SVD transmission.

DSL vectoring was proposed in [4] with the objective of indomain FEXT cancellation. Also, the DSL multiuser theory given in [11] describes the use of the GDFE for the purpose of noise decorrelation.

The analysis of this section is based on the GDFE formulation. Starting from (15), the feedforward operation of the GDFE is expressed as

$$
\begin{aligned}
\mathbf{Z}_{n}^{\prime} & =G_{n}^{-1} \mathbf{Z}_{n} \\
& =G_{n}^{-1} T_{n} \mathbf{W}_{n}+\mathbf{E}_{n},
\end{aligned}
$$

where $\mathbf{Z}_{n}^{\prime}$ is the output of the operation, $G_{n}$ is as given in (16), and $\mathbf{E}_{n}$ is the resulting error term with $R_{\mathbf{E}_{n} \mathbf{E}_{n}}=I$. Although the feedforward operation decorrelates the noise, it introduces interference among the in-domain pairs, since $G_{n}^{-1} T_{n}$ is nondiagonal. To eliminate this interference, a feedback operation is applied,

$$
\begin{aligned}
\widetilde{\mathbf{Z}}_{n} & =\mathbf{Z}_{n}^{\prime}-\left(G_{n}^{-1} T_{n}-D_{n}\right) \widehat{\mathbf{W}}_{n} \\
& =\mathbf{Z}_{n}^{\prime}-F_{n} \widehat{\mathbf{W}}_{n} \\
& =T_{n} \mathbf{W}_{n}+\mathbf{E}_{n},
\end{aligned}
$$




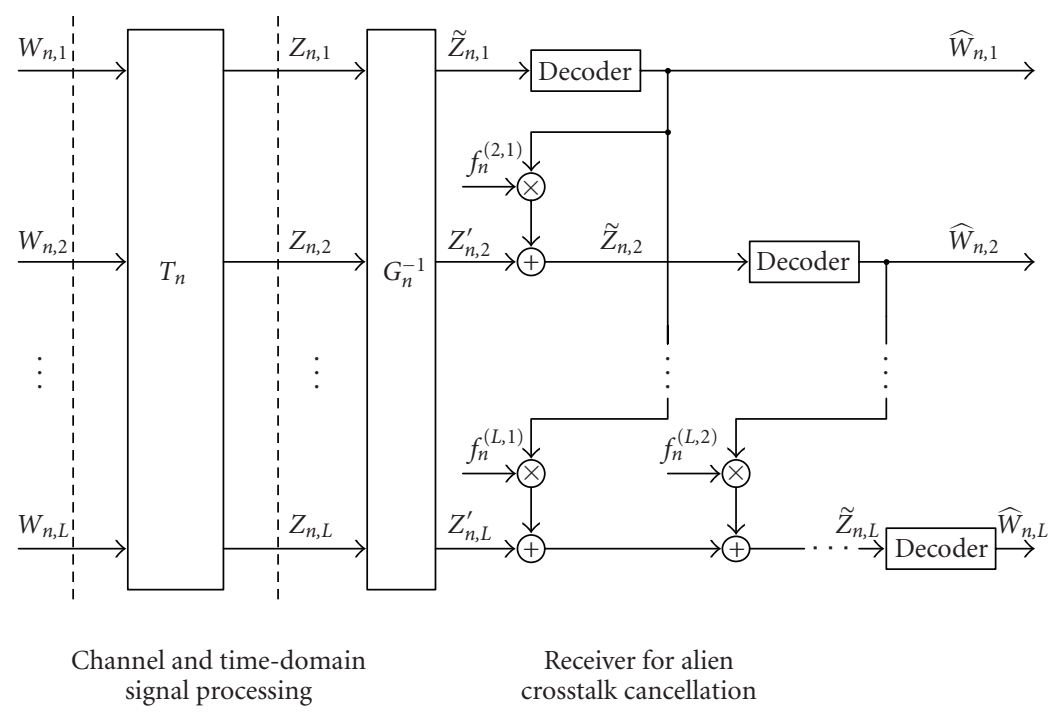

Figure 9: Generalized decision feedback.

where $\widetilde{\mathbf{Z}}_{n}$ is the output of the operation, and $D_{n}$ is a diagonal matrix whose elements are the diagonal elements of $G_{n}^{-1} T_{n}$. The vector $\widehat{\mathbf{W}}_{n}$ represents the decoded symbols, for which it is here assumed that they are always correct.

The transmitter and receiver structures are shown in Figure 9.

Next, the mutual information is computed. The mutual information of the original channel can be expressed as

$$
\begin{aligned}
I\left(\mathbf{W}_{n} ; \mathbf{Z}_{n}\right) & =I\left(\mathbf{W}_{n} ; G_{n} \mathbf{Z}_{n}^{\prime}\right) \\
& =I\left(\mathbf{W}_{n} ; \mathbf{Z}_{n}^{\prime}\right) \\
& =H\left(\mathbf{Z}_{n}^{\prime}\right)-H\left(\mathbf{Z}_{n}^{\prime} \mid \mathbf{W}_{n}\right) \\
& =H\left(\mathbf{Z}_{n}^{\prime}\right)-H\left(\mathbf{Z}_{n}^{\prime}-F_{n} \mathbf{W}_{n} \mid \mathbf{W}_{n}\right) \\
& =I\left(\mathbf{W}_{n} ; \mathbf{Z}_{n}^{\prime}-F_{n} \mathbf{W}_{n}\right) \\
& =I\left(\mathbf{W}_{n} ; \widetilde{\mathbf{Z}}_{n}\right),
\end{aligned}
$$

where (31) holds because multiplication by $G_{n}$ represents a 1-1 transformation, and (34) is obvious by the definition of conditional entropy [21]. The above formulas show that the GDFE scheme does not reduce the mutual information of the channel.

Finally, a simple expression is given for the mutual information based on (30):

$$
\begin{aligned}
I\left(\mathbf{W}_{n} ; \tilde{\mathbf{Z}}_{n}\right) & =\frac{1}{2} \log _{2}\left(\frac{\left|T_{n} R_{\mathbf{W}_{n} \mathbf{W}_{n}} T_{n}^{*}+R_{\mathbf{E}_{n} \mathbf{E}_{n}}\right|}{\left|R_{\mathbf{E}_{n} \mathbf{E}_{n}}\right|}\right) \\
& \leq \sum_{k=1}^{L} \frac{1}{2} \log _{2}\left(1+\frac{\left|\tau_{n, k}\right|^{2} \boldsymbol{E}_{n, k}}{\sigma_{n, k}^{2}}\right),
\end{aligned}
$$

where $\tau_{n, k}$ are the diagonal elements of $T_{n}, \mathcal{E}_{n, k}$ are the diagonal elements of $R_{\widetilde{\mathbf{W}}_{n} \widetilde{\mathbf{W}}_{n}}$, and $\sigma_{n, k}^{2}$ are the diagonal elements of $R_{\mathrm{E}_{n} \mathrm{E}_{n}}$. The inequality relation follows from Hadamard's inequality, and equality holds when the off-diagonal elements of $R_{\widetilde{\mathbf{W}}_{n} \widetilde{\mathbf{W}}_{n}}$ equal 0 . Thus, the maximization of the sum capacity can be achieved by solving the power allocation problem on parallel channels.
A note on the decoding order of the vectored pairs can be made. It can be observed that changing the decoding order is equivalent to premultiplying $\mathbf{W}_{n}$ and $\mathbf{Z}_{n}$ by a permutation matrix. Such an operation indeed changes the values of $\tau_{n, k}$ and $\sigma_{n, k}$, however, it is easy to see that the mutual information as defined above is not affected. This implies that the decoding order affects the rate of each pair but not the highest possible sum rate.

\subsection{Receiver coordination with noise-prediction structure}

An alternative to the decision-feedback structure is next described. Instead of decorrelating the noise with the feedforward section and then cancelling the interference by subtracting weighted estimates of the symbols, the receiver noise can be directly decorrelated by estimating and subtracting the error of the decoder. This is similar to the well-known concept of a noise-predictive decision-feedback equalizer [23].

From (16), the noise vector is expressed as

$$
\mathbf{N}_{n}=G_{n} \mathbf{E}_{n}
$$

where $\mathbf{E}_{n}$ is called the innovations vector. The lower-triangular property of the $G_{n}$ matrix naturally leads to the following noise decorrelation procedure. First, $N_{n, 1}=E_{n, 1}$, which is easily found by subtracting the decoder output of pair 1 from the decoder input of pair 1 . Then,

$$
\begin{aligned}
N_{n, 2} & =E_{n, 2}+g_{n}^{(2,1)} E_{n, 1} \Longleftrightarrow E_{n, 2} \\
& =N_{n, 2}-g_{n}^{(2,1)} E_{n, 1},
\end{aligned}
$$

where $g_{n}^{(2,1)}$ is the element of $G_{n}$ at row 2 and column 1. So, the noise term of pair 2 can be decorrelated by subtracting $g_{n}^{(2,1)} E_{n, 1}$ from the received signal of pair 2 . Then, $E_{n, 2}$ is estimated by subtracting the decoder output of pair 2 from 


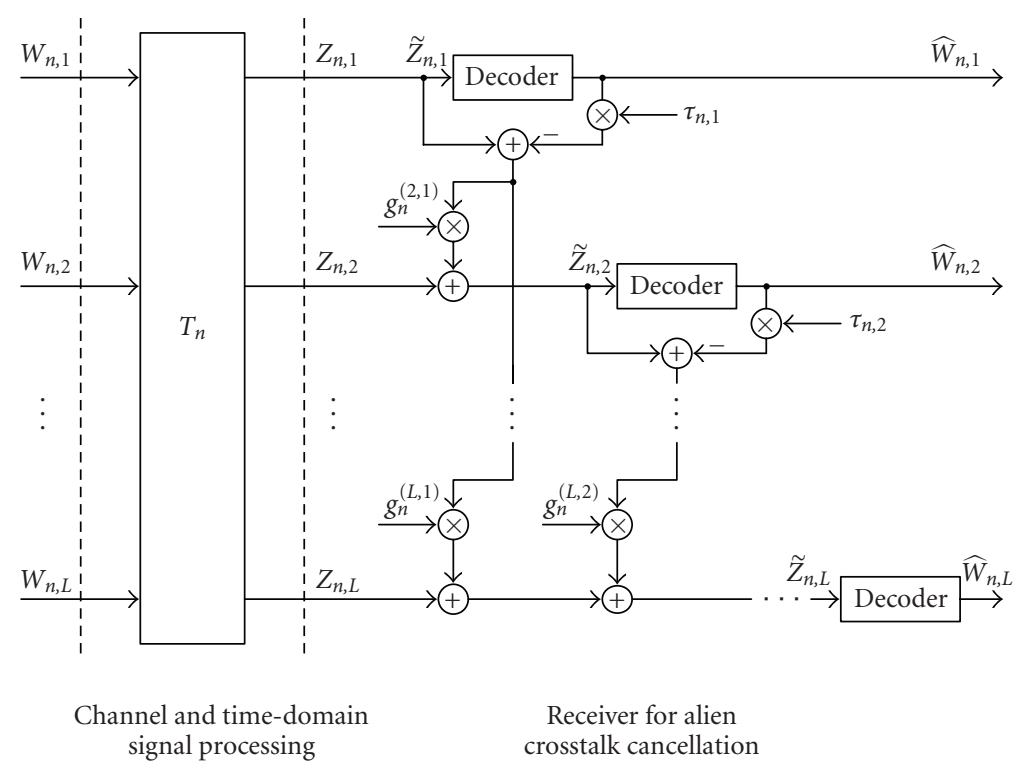

FIgURE 10: Error whitening.

the decoder input of pair 2. This process continues for the remaining pairs, where in each iteration the previously estimated errors $E_{n, 1}, \ldots, E_{n, k-1}$ are weighted and subtracted from the received signal of $k$.

Starting with (15), the noise-prediction operation is expressed as

$$
\begin{aligned}
\widetilde{\mathbf{Z}}_{n} & =\mathbf{Z}_{n}+\left(I-G_{n}\right) \mathbf{E}_{n} \\
& =T_{n} \mathbf{W}_{n}+\mathbf{N}_{n}+\left(I-G_{n}\right) \mathbf{E}_{n} \\
& =T_{n} \mathbf{W}_{n}+\mathbf{E}_{n},
\end{aligned}
$$

where it is seen that the resulting noise term is uncorrelated, and $T_{n}, \mathbf{E}_{n}$ are the same as in (30).

The transmitter and receiver structures are shown in Figure 10.

An interesting advantage of this scheme is the lack of the feedforward section. It should be noted that there are several possibilities for the computation of the error $\mathbf{E}_{n}$. The DSL decoder consists of multiple stages, and the error computation can be computed based on the output of any of these stages. The typical DSL decoder includes an inner decoder (e.g., trellis decoder) and an outer decoder (e.g., Reed-Solomon decoder). The error can be found by comparing the symbols at the decoder input with the estimated symbols corresponding to either the outer or the inner decoder. The error can even be estimated by obtaining a decision through a "slicer" operation. In each of these cases, there is a tradeoff between latency and error propagation.

Since the expressions of the equivalent channels in (30) and (43) are identical, it is clear that

$$
I\left(\mathbf{W}_{n} ; \mathbf{Z}_{n}\right)=I\left(\mathbf{W}_{n} ; \tilde{\mathbf{Z}}_{n}\right),
$$

which implies that the noise-prediction scheme has no information loss compared to the DFE scheme, or compared to the original channel.
For the purpose of gaining intuition, an alternative approach is here used to arrive at (38). Using the chain rule, the mutual information of the channel is expressed as

$$
I\left(\mathbf{W}_{n} ; \mathbf{Z}_{n}\right)=\sum_{k=1}^{L} I\left(W_{n, k} ; \mathbf{Z}_{n} \mid W_{n, 1}, \ldots, W_{n, k-1}\right) .
$$

A second use of the chain rule on each individual term of the above sum yields

$$
\begin{aligned}
& I\left(W_{n, k} ; \mathbf{Z}_{n} \mid W_{n, 1}, \ldots, W_{n, k-1}\right) \\
& \quad=\sum_{m=1}^{L} I\left(W_{n, k} ; Z_{n, m} \mid W_{n, 1}, \ldots, W_{n, k-1}, Z_{n, 1}, \ldots, Z_{n, m-1}\right) .
\end{aligned}
$$

The terms of this sum are next individually investigated. For $m<k$,

$$
\begin{aligned}
I\left(W_{n, k} ;\right. & \left.Z_{n, m} \mid W_{n, 1}, \ldots, W_{n, k-1}, Z_{n, 1}, \ldots, Z_{n, m-1}\right) \\
= & H\left(Z_{n, m} \mid W_{n, 1}, \ldots, W_{n, k-1}, Z_{n, 1}, \ldots, Z_{n, m-1}\right) \\
& -H\left(Z_{n, m} \mid W_{n, 1}, \ldots, W_{n, k-1}, W_{n, k}, Z_{n, 1}, \ldots, Z_{n, m-1}\right) \\
= & H\left(E_{n, m} \mid W_{n, 1}, \ldots, W_{n, k-1}, Z_{n, 1}, \ldots, Z_{n, m-1}\right) \\
& -H\left(E_{n, m} \mid W_{n, 1}, \ldots, W_{n, k-1}, W_{n, k}, Z_{n, 1}, \ldots, Z_{n, m-1}\right) \\
= & 0,
\end{aligned}
$$

where (49) follows from the fact that $E_{n, m}$ is the only variable term of $Z_{n, m}$ given the conditions, and (50) holds because the conditioning on $W_{n, k}$ provides no additional information 
about $E_{n, m}$. For $m>k$,

$$
\begin{aligned}
& I\left(W_{n, k} ; Z_{n, m} \mid W_{n, 1}, \ldots, W_{n, k-1}, Z_{n, 1}, \ldots, Z_{n, m-1}\right) \\
& =H\left(W_{n, k} \mid W_{n, 1}, \ldots, W_{n, k-1}, Z_{n, 1}, \ldots, Z_{n, m-1}\right) \\
& \quad-H\left(W_{n, k} \mid W_{n, 1}, \ldots, W_{n, k-1}, W_{n, k}, Z_{n, 1}, \ldots, Z_{n, m-1} Z_{n, m}\right)
\end{aligned}
$$

$$
=0 \text {, }
$$

where (53) holds because the conditioning on $Z_{n, m}$ offers no additional information on $W_{n, k}$. Then, for $m=k$,

$$
\begin{aligned}
& I\left(W_{n, k} ; Z_{n, k} \mid W_{n, 1}, \ldots, W_{n, k-1}, Z_{n, 1}, \ldots, Z_{n, k-1}\right) \\
& =I\left(W_{n, k} ; Z_{n, k}\right. \\
& \left.\quad-\sum_{m=1}^{k-1} f_{n}^{(k, m)} E_{n, m} \mid W_{n, 1}, \ldots, W_{n, k-1}, Z_{n, 1}, \ldots, Z_{n, k-1}\right)
\end{aligned}
$$

$$
=I\left(W_{n, k} ; \tilde{Z}_{n, k}\right)
$$

where $f_{n}^{(k, m)}$ is the element of $F_{n}$ on row $k$ and column $m$, and (55) follows from the fact that the conditioning fully determines $E_{n, m}$ for $m=1, \ldots, k-1$. Thus, it is found that

$$
\begin{aligned}
I\left(\mathbf{W}_{n} ; \mathbf{Z}_{n}\right) & =\sum_{k=1}^{L} I\left(W_{n, k} ; \tilde{Z}_{n, k}\right) \\
& \leq \sum_{k=1}^{L} \frac{1}{2} \log _{2}\left(1+\frac{\left|\tau_{n, k}^{2}\right| \mathcal{E}_{n, k}}{\sigma_{n, k}^{2}}\right) .
\end{aligned}
$$

Again, it is noted that changing the decoding order affects the rate of each individual pair, but does not change the sum rate.

\section{SIMULATION RESULTS}

In this section, simulation results are shown to demonstrate the performance benefits that can be realized with alien noise cancellation. These are generated by "frequency-domain" simulations, where the SNR is computed for each tone by using the knowledge of the channel and of the transmitted PSD, and by evaluating the receiver noise level. The SNR per tone is then converted to the number of bits per tone, which leads to an estimate of the achievable data rate. In the following, the simulation assumes the error-whitening structure of Figure 10. But, as noted in Section 3, the maximum achievable data-rate sum over all vectored pairs is the same regardless of the specific noise decorrelation method.

Similarly to the example of Section 2.4, downstream communication is considered, where the vectored system consists of two pairs and there is a single T1 disturber (upstream and downstream) that causes interference. Again, the vectored pairs employ ADSL2+ for communication and use the PSD mask of Annex A [18]. The NEXT coupling coefficients and the T1 PSD are the same as in the example. Additionally, FEXT coupling from the T1 disturber to the pairs is taken into account. The FEXT coupling was obtained from

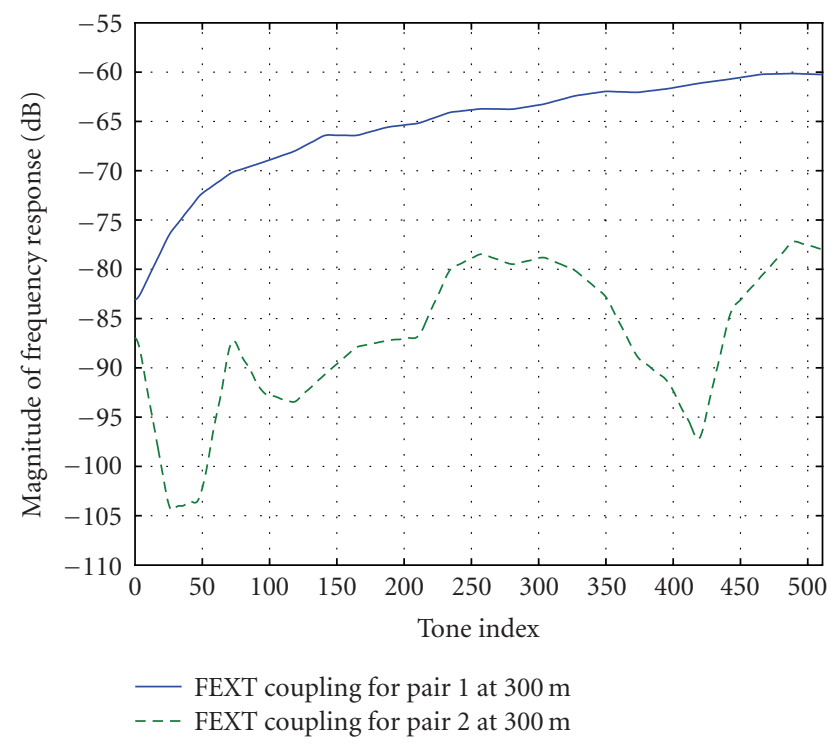

FIGURE 11: FEXT coupling for the 2 vectored pairs at $300 \mathrm{~m}$.

TABLE 1: Simulation parameters.

\begin{tabular}{lc}
\hline Number of DMT tones & 512 \\
Tone width & $4.3125 \mathrm{kHz}$ \\
Symbol rate & $4 \mathrm{kHz}$ \\
Coding gain & $6 \mathrm{~dB}$ \\
Noise margin & $6 \mathrm{~dB}$ \\
SNR gap & $9.8 \mathrm{~dB}$ \\
Maximum power & $20.4 \mathrm{dBmW}$ \\
Cable type & $26-\mathrm{Gauge}$ \\
Source/load impedance & $100 \mathrm{Ohm}$ \\
Max information bits per tone & 14 \\
Background noise & $-140 \mathrm{dBmW} / \mathrm{Hz}$ \\
\hline
\end{tabular}

measurements obtained with the same setup as that for the NEXT measurements. The magnitude of the FEXT coupling is shown in Figure 11. Since these measurements were obtained with a $300 \mathrm{~m}$ loop, the FEXT coupling was normalized for other loop lengths using the well-known rule according to which the FEXT PSD is proportional to the loop length and to the squared magnitude of the channel transfer function. Note that no in-domain crosstalk has been included in the simulation. The rest of the simulation assumptions are shown in Table 1.

Figure 12 shows the noise PSDs of the two receivers for a $3 \mathrm{~km}$ loop. The noise of pair 1 is unaffected by the noise decorrelation algorithm. The noise of pair 2 is significantly mitigated after the application of the algorithm. Note that the frequencies where the noise of pair 2 is not greatly reduced correspond to those frequencies where pairs 1 and 2 have weak noise correlation.

Next, Figure 13 shows the bit distributions of the two receivers for the same case. As expected, the bit distribution of pair 1 is unchanged, but pair 2 is capable of transmitting 


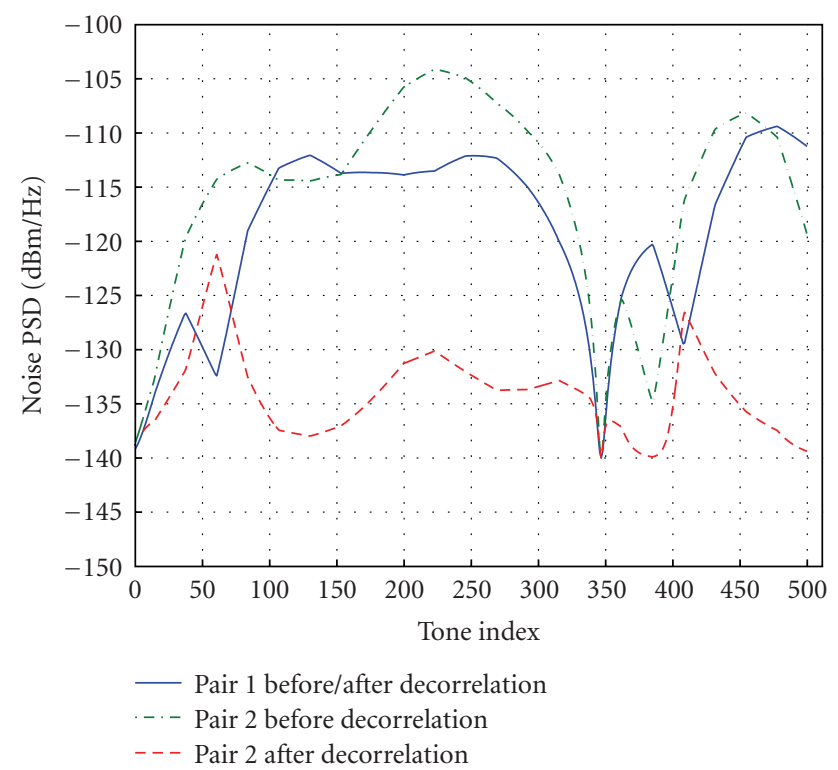

FIGURE 12: Noise PSD before and after noise decorrelation with $3000 \mathrm{~m}$ loop.

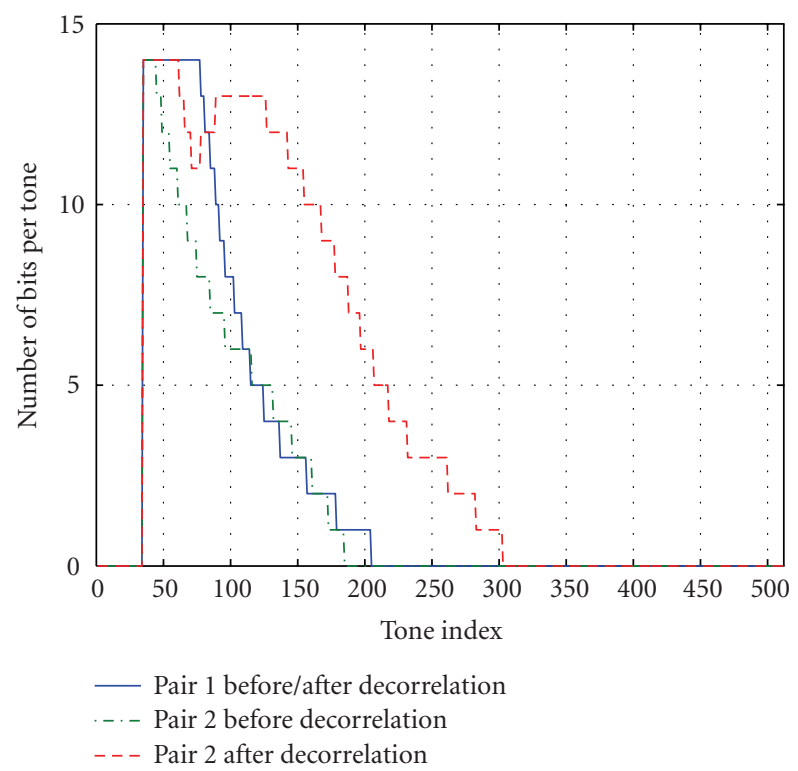

FIgURE 13: Bit distributions before and after noise decorrelation with 3000 m loop.

more bits per tone after noise decorrelation as a result of the higher receiver SNR.

Finally, Figure 14 shows the rate-reach curves of the 2 pairs and also the sum rate for loops between 1 and $4 \mathrm{~km}$. Of course, the rate-reach curve of pair 1 is the same before and after noise decorrelation, but the rate increase of pair 2 is significant over almost all loop lengths. In the shortest loops, the improvements become smaller mainly because of the cap on the number of bits that can be transported on each tone.

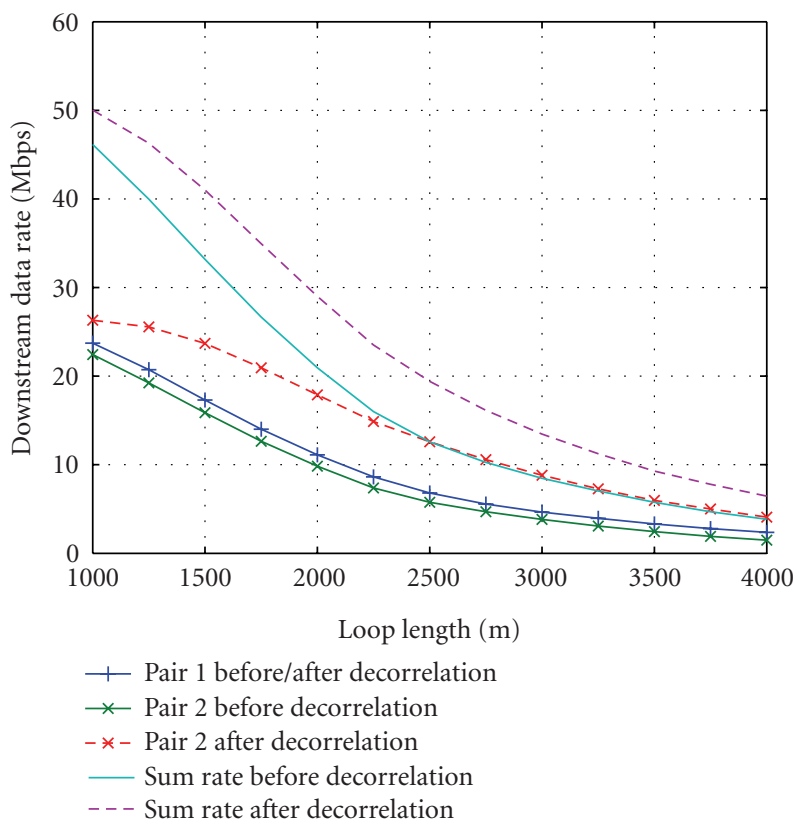

FIGURE 14: Rate-reach curves before and after noise decorrelation.

Performance gains over a wide range of loops have also been observed with other NEXT interferers that affect a significant portion of the downstream or upstream band. For FEXT interferers (e.g., FEXT from a similar system), the gains are considerable primarily in short enough loops, where FEXT is the dominant noise source.

\section{CONCLUSIONS}

This paper gave an overview of the subject of alien crosstalk cancellation for DSL systems employing multiple pairs. First, it was shown that a common interference source leads to noise correlation among the victim DSL pairs, and that such correlation is strongest within the same tones. Then, specific methods were presented to perform crosstalk cancellation by exploiting the noise correlation property. It was theoretically shown that the methods have equivalent performance with respect to the sum rate over the pairs. Last, performance simulation results were presented to illustrate the potential benefits.

It is worth making some additional comments about the practical application of noise decorrelation. Although not presented in this paper, the noise decorrelation methods require the existence of additional algorithms for initialization (before the DSL link is established) and for adaptation (after the DSL link is operational). The initialization algorithms aim at producing estimates of the noise correlation, which was simply assumed to be known in advance in this paper. The adaptation (or updating) algorithms are needed since it is generally expected that crosstalk interference varies over time, either due to slow-varying variations of the crosstalk coupling, or due to crosstalk sources becoming active or inactive.

Finally, the results presented in [24] demonstrated that in-domain crosstalk cancellation can be simplified by careful 
choice of the tones and pairs over which joint signal processing is applied. This previous conclusion indicates that similar selection methods can also be applied for alien crosstalk cancellation, thus offering the possibility of complexity reduction.

\section{ACKNOWLEDGMENT}

The authors would like to thank Bin Lee of Stanford University for providing the crosstalk measurements.

\section{REFERENCES}

[1] M. L. Honig, K. Steiglitz, and B. Gopinath, "Multichannel signal processing for data communications in the presence of crosstalk," IEEE Transactions on Communications, vol. 38, no. 4, pp. 551-558, 1990.

[2] J. W. Lechleider, "Coordinated transmission for two-pair digital subscriber lines," IEEE Journal on Selected Areas in Communications, vol. 9, no. 6, pp. 920-930, 1991.

[3] J. Huber and R. Fischer, "Dynamically coordinated reception of multiple signals in correlated noise," in Proceedings of IEEE International Symposium on Information Theory (ISIT '94), pp. 132-132, Trondheim, Norway, June-July 1994.

[4] G. Ginis and J. M. Cioffi, "Vectored-DMT: a FEXT canceling modulation scheme for coordinating users," in Proceedings of IEEE International Conference on Communications (ICC '01), vol. 1, pp. 305-309, Helsinki, Finland, June 2001.

[5] G. Ginis and J. M. Cioffi, "Vectored transmission for digital subscriber line systems," IEEE Journal on Selected Areas in Communications, vol. 20, no. 5, pp. 1085-1104, 2002.

[6] G. Tauböck and W. Henkel, "MIMO systems in the subscriberline network," in Proceedings of 5th International OFDMWorkshop (InOWo '00), pp. 18.1-18.3, Hamburg, Germany, September 2000.

[7] R. Cendrillon, G. Ginis, M. Moonen, K. Van Acker, T. Bostoen, and P. Vandaele, "Partial crosstalk precompensation in downstream VDSL," Signal Processing, vol. 84, no. 11, pp. 20052019, 2004.

[8] R. Cendrillon, M. Moonen, G. Ginis, K. Van Acker, T. Bostoen, and P. Vandaele, "Partial crosstalk cancellation for upstream VDSL," EURASIP Journal on Applied Signal Processing, vol. 2004, no. 10, pp. 1520-1535, 2004.

[9] R. Cendrillon, M. Moonen, E. Van den Bogaert, and G. Ginis, "The linear zero-forcing crosstalk canceller is near-optimal in DSL channels," in Proceedings of IEEE Global Telecommunications Conference (GLOBECOM '04), vol. 4, pp. 2334-2338, Dallas, Tex, USA, November-December 2004.

[10] R. Cendrillon, G. Ginis, M. Moonen, J. Verlinden, and T. Bostoen, "Improved linear crosstalk precompensation for DSL," in Proceedings of IEEE International Conference on Acoustics, Speech, Signal Processing (ICASSP '04), vol. 4, pp. 1053-1056, Montreal, Quebec, Canada, May 2004.

[11] T. Starr, M. Sorbara, J. M. Cioffi, and P. J. Silverman, DSL Advances, Prentice-Hall, Englewood Cliffs, NJ, USA, 2003.

[12] T. Magesacher, P. Ödling, P. O. Börjesson, et al., "On the capacity of the copper cable channel using the common mode," in Proceedings of IEEE Global Telecommunications Conference (GLOBECOM '02), vol. 2, pp. 1269-1273, Taipei, Taiwan, Novemeber 2002.

[13] M. Tsatsanis, "Vectoring techniques for multi-line 10MDSL systems," T1E1.4 committee, contribution 2002-196, August 2002.
[14] K. Schneider and L. Sandstrom, "MIMO vs. SISO capacity on twisted pair loops," T1E1.4 committee, contribution 2002-259, November 2002.

[15] M. Tsatsanis, M. A. Erickson, and S. Shah, "Multiline transmission in communication systems," International application published under the Patent Cooperation Treaty, international application number PCT/US03/18004.

[16] F. Sjöberg, M. Isaksson, R. Nilsson, P. Ödling, S. K. Wilson, and P. O. Börjesson, "Zipper: a duplex method for VDSL based on DMT," IEEE Transactions on Communications, vol. 47, no. 8, pp. 1245-1252, 1999.

[17] ITU-T Recommendation G.993.1, Very high speed digital subscriber line, June 2004.

[18] ITU-T Recommendation G.992.5, Asymmetrical Digital Subscriber Line (ADSL) transceivers-Extended bandwidth ADSL2 (ADSL2plus+), May 2003.

[19] G. G. Raleigh and J. M. Cioff, "Spatio-temporal coding for wireless communication," IEEE Transactions on Communications, vol. 46, no. 3, pp. 357-366, 1998.

[20] J. M. Cioffi and G. D. Forney Jr., "Generalized decisionfeedback equalization for packet transmission with ISI and Gaussian noise," in Communications, Computation, Control, and Signal Processing: A Tribute to Thomas Kailath, A. Paulraj, V. P. Roychowdhury, and C. D. Schaper, Eds., chapter 4, pp. 79-127, Kluwer Academic, Boston, Mass, USA, 1997.

[21] T. M. Cover and J. A. Thomas, Elements of Information Theory, John Wiley \& Sons, New York, NY, USA, 1991.

[22] S. Kasturia, J. T. Aslanis, and J. M. Cioffi, "Vector coding for partial response channels," IEEE Transactions on Information Theory, vol. 36, no. 4, pp. 741-762, 1990.

[23] J. G. Proakis, Digital Communications, McGraw-Hill, New York, NY, USA, 3rd edition, 1995.

[24] R. Cendrillon, M. Moonen, G. Ginis, K. Van Acker, T. Bostoen, and P. Vandaele, "Partial crosstalk cancellation exploiting line and tone selection in upstream VDSL," in Proceedings of 6th Baiona Workshop on Signal Processing in Communications (Baiona '03), Baiona, Spain, September 2003.

George Ginis received the Diploma in electrical and computer engineering from the National Technical University of Athens, Athens, Greece, in 1997, and the M.S. and Ph.D. degrees in electrical engineering from Stanford University, Stanford, Calif, in 1998 and 2002, respectively. He is currently a Member Group Technical Staff with DSP Systems of Texas Instruments Incoporated. His research interests include mul-

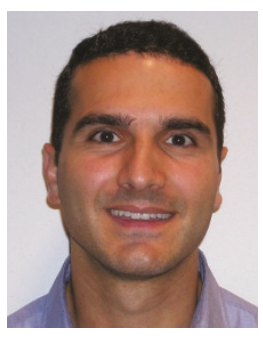
tiuser transmission theory, interference mitigation, and their applications to wireline and wireless communications.

Chia-Ning Peng was born in Taipei, Taiwan, in 1968. He received his B.S. degree in electrical engineering from National Taiwan University in 1990 and the M.S. and Ph.D. degrees in electrical engineering from the University of Michigan, Ann Arbor, Mich, USA, in 1994 and 1998, respectively. His research interests include error-correction codes, digital communications theory and application, and applied signal processing.

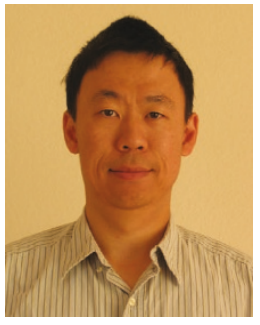

He now works for Texas Instruments Incorporated in the area of residential gateways. 